\title{
A writer identification and verification system using HMM based recognizers
}

\author{
Andreas Schlapbach $\cdot$ Horst Bunke
}

Received: 12 November 2004 / Accepted: 6 July 2006/Published online: 6 October 2006

(C) Springer-Verlag London Limited 2006

\begin{abstract}
In this paper, an off-line, text independent system for writer identification and verification of handwritten text lines using Hidden Markov Model (HMM) based recognizers is presented. For each writer, an individual recognizer is built and trained on text lines of that writer. This results in a number of recognizers, each of which is an expert on the handwriting of exactly one writer. In the identification and verification phase, a text line of unknown origin is presented to each of these recognizers and each one returns a transcription that includes the log-likelihood score for the generated output. These scores are sorted and the resulting ranking is used for both identification and verification. Several confidence measures are defined on this ranking. The proposed writer identification and verification system is evaluated using different experimental setups.
\end{abstract}

Keywords Writer identification - Writer verification . Off-line handwriting · HMM based handwriting recognition

\section{Introduction}

In recent years, significant progress has been made in recognizing a person based on biometric features [13-15]. Prominent biometric modalities include face,

\footnotetext{
A. Schlapbach $(\bowtie) \cdot$ H. Bunke

Institut für Informatik und angewandte Mathematik,

Universität Bern, Neubrückstrasse 10,

3012 Bern, Switzerland

e-mail: schlpbch@iam.unibe.ch

H. Bunke

e-mail: bunke@iam.unibe.ch
}

fingerprint, iris, signature, and voice. In this paper we address the problem of personal identification and verification based on a person's handwriting. Writer identification is the task of determining the author of a sample handwriting from a set of writers [22]. Related to this task is writer verification, i.e., the task of determining whether or not a handwritten text has been written by a certain person. If any text may be used to establish the identity of the writer the task is text independent. Otherwise, if a writer has to write a particular predefined text-such as his or her signature-to identify himself or herself, or to verify his or her identity, the task is text dependent. Writer identification and verification can be performed on-line, where temporal and spatial information about the writing is available, or off-line, where only a scanned image of the handwriting is available. The system we propose in this paper performs text independent writer identification and verification using off-line handwritten text lines. Compared to other works which base their approach on either single words or on whole pages of handwritten text (see Sect. 2 for a discussion of related work), we use text lines as basic input units. Examples of handwritten text lines from our database, produced by different writers, are given in Fig. 1 . Possible applications of our system include forensic writer identification [31], the retrieval of handwritten documents from a database [2, 3], or authorship determination of historical manuscripts.

For both isolated word and general text recognition, Hidden Markov Models (HMMs) have become the predominant approach. In fact, HMM based recognizers have a number of advantages over other approaches [9]. First, they are resistant to noise and can cope with shape variations. Secondly, HMM based 
In the first place it is not a great deal

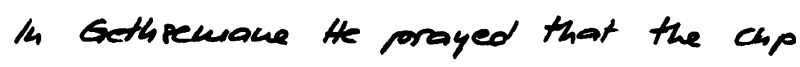

Amen. MOST people would prolably regard tiredness as a

Nor is she necessarity heing deceitful. She really did feel tired

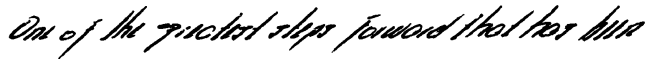

As it is, with so much of our life already

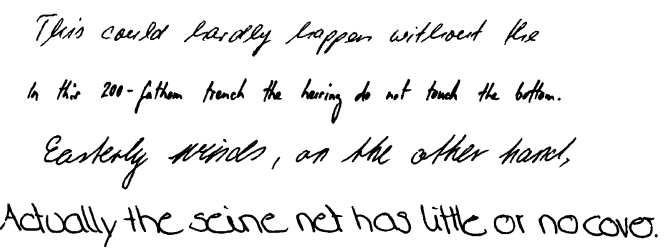

Fig. 1 Examples of text lines used for writer identification and verification

recognizers are able to implicitly segment a text line into words and characters, a task that is difficult to perform explicitly [32]. Thirdly, there exist standard algorithms for training and testing [23].

This paper is based on the idea of utilizing an existing HMM-based handwriting recognition system, which has been optimized for text recognition, for the purpose of writer identification and verification. For each writer in the considered population, an individual HMM based handwriting recognition system is trained using only data from that writer. Thus for $n$ different writers we obtain $n$ different HMMs. They all have the same architecture, but their parameters, i.e., transition and output probabilities, are different because they are trained on different data each. Intuitively, each HMM can be understood as an expert specialized in recognizing the handwriting of one particular person. Given an arbitrary line of text as input, each HMM based recognizer outputs a transcription of the input together with a recognition score. These outputs are sorted in decreasing order of the recognition scores, producing a ranking of all systems. Assuming that correctly recognized words have a higher score than incorrectly recognized ones, and assuming furthermore that the recognition rate of a system is higher on input from the writer the system is trained on than on input from other writers, we can utilize the scores produced by the different HMMs for the task of identifying the writer of a text line or verifying whether a text line has actually been written by the person who claims to be the writer.

This paper is structured as follows. In the next section, we present related work. In Sect. 3 our system for handwritten text line identification and verification is introduced. Section 4 presents experimental results on the identification as well as on the verification task, and Sect. 5 concludes the paper.

\section{Related work}

Surveys covering work in automatic writer identification and signature verification until 1993 are given in $[16,22]$. Writer identification can be understood as a classification problem where a word, text fragment, or text is to be assigned to one out of a number of possible writers. Recently, different approaches to writer identification have been proposed. Said et al. [25] treat the writer identification task as a texture analysis problem. They use global statistical features extracted from the entire image of a text using multi-channel Gabor filtering and grey-scale co-occurrence matrix techniques.

Srihari et al. [10,34] address the problem of writer verification, i.e., the problem of determining whether two documents are written by the same person or not. In order to identify the writer of a given document, they model the problem as a classification problem with two classes, authorship and non-authorship. Given two handwriting samples, one of known and the other of unknown identity, the distance between two documents is computed. Then the distance value is used to classify the data as positive or negative.

Zois et al. [35] base their approach on single words by morphologically processing horizontal projection profiles. The projections are partitioned into a number of segments from which feature vectors are extracted. A Bayesian classifier and a neural network are then applied to the feature vectors.

In Hertel et al. [12] a system for writer identification is described. The system first segments a given text into individual text lines and then extracts a set of features from each text line. The features are subsequently used in a $k$-nearest-neighbor classifier that compares the feature vector extracted from a given input text to a number of prototype vectors coming from writers with known identity.

Bulacu et al. [8] use edge-based directional probability distributions as features for the writer identification task. The authors introduce edge-hinge distribution as a new feature. The key idea behind this feature is to consider two edge fragments in the neighborhood of a pixel and compute the joint probability distribution of the orientations of the two fragments. Additionally, in [29] as a new feature the histogram of connected-component contours $\left(\mathrm{CO}^{3}\right)$ for upper-case handwriting is introduced. Combining this feature with the edge-hinge feature achieves better results than each of the features used separately. In 
[30] this approach is extended to mixed-style handwriting using fragmented connected-component contours $\left(\mathrm{FCO}^{3}\right)$.

In a number of papers $[4,5,21]$ graphemes are proposed as features for describing the individual properties of handwriting. Furthermore, it is shown that each handwriting can be characterized by a set of invariant features called the writer's invariants. These invariants are detected using an automatic grapheme clustering procedure. In [6] these graphemes are used to address the writer verification task based on text blocks as well as on handwritten words.

Leedham et al. [17] present a set of eleven features which can be extracted easily and used for the identification and verification of documents containing handwritten digits. These features are represented as vectors, and by using the Hamming distance measure and determining a threshold value for the intra-author variation a high degree of accuracy in authorship detection is achieved.

The idea of using HMM based recognizers for the purpose of writer identification was first proposed in [26] and a preliminary version of the paper appeared in [27] which also addresses the task of writer verification. The current paper has been significantly extended with respect to methodology and experimental evaluation. We also provide an analysis of the relationship between writer identification and text recognition rate.

\section{Writer identification and verification using HMM based recognizers}

In this paper a system for the identification and verification of handwritten text lines using HMM based recognizers is presented. A schematic overview of the system is shown in Fig. 2. For each writer, a text line recognizer as described in Sect. 3.1 is built and trained with data coming from this writer only. As a result of the training procedure, we get a recognizer for each writer that is an expert on the handwriting style of that particular writer. Presented with a text line of unknown identity each recognizer returns a transcription containing the log-likelihood score of the text line. These scores are sorted and based on the resulting ranking writer identification (see Sect. 3.2) or writer verification (see Sect. 3.3) are performed.

\subsection{HMM based recognizer}

Our system uses an HMM based recognizer that has been designed and optimized for the task of handwritten text line recognition. Except for some nor-

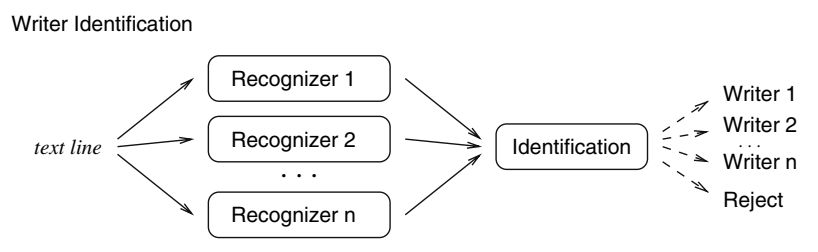

Writer Verification

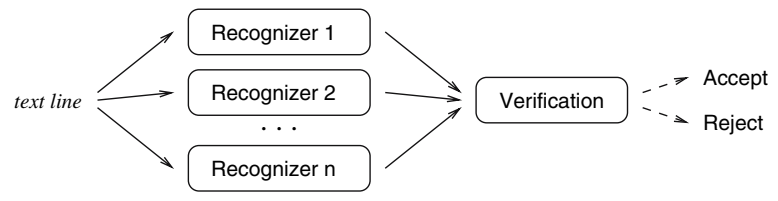

Fig. 2 Schematic overview of the writer identification and verification system

malization operations (see next two paragraphs), this recognizer is treated as a black box and used without any modification. For the purpose of completeness, we include a brief description of this recognizer here. More details can be found in [18].

In a first preprocessing step, the image is contrast enhanced. Next, the skew of a text line is corrected such that the text line is aligned horizontally. These normalization operations do not remove any writer specific information.

The other normalization operations we apply have been determined emiprically. In a previous paper [28], we have studied the effect of normalization operations on the identification rate of our system. The operations considered are slant correction, width normalization and vertical scaling. A detailed description of the normalization operations is given in [18]. On the one hand, there exists a strong correlation between the text recognition and the writer identification rate, and applying normalization operations increases the text recognition rate. On the other hand, normalization also removes writer-specific information from a handwritten text line. Hence there is a trade-off between optimizing the text recognition performance of our system and keeping writer specific features. Our experiments have shown that the highest writer identification rates are obtained if slant correction and width normalization are omitted and only vertical scaling is applied. Consequently, we perform vertical scaling, in the same way as described in [18], but do not apply slant correction and width normalization. In Fig. 3 a text line before and after normalization is shown.

A sliding window of one pixel width moves over the normalized text line from left to right and extracts nine features, three global and six local ones. The three global features are the fraction of black pixels in the window, the center of gravity and the second order moment, while the six local features represent the 


$$
\begin{aligned}
& \text { By the end of the menth he still delighted in Noples. He } \\
& \text { Text line before normalization. } \\
& \text { By the end of the menth he still delighted in Noples. He } \\
& \text { Text line after normalization. }
\end{aligned}
$$

Fig. 3 A text line before and after normalization

position and orientation of the upper- and the lowermost pixel, the number of black-to-white transitions in the window, and the fraction of black pixels between the upper- and the lower-most black pixel. Using these nine features, an input text line is converted into a sequence of nine-dimensional feature vectors.

This set of features is not inspired by forensic research but forms an abstract representation of a person's handwriting and has shown good performance in handwritten text recognition [18]. The features extracted from a text line are tested for Roman writing only. Their suitability for non Roman writing styles is an interesting, open question beyond the scope of the current paper.

The characters of the text lines are modeled using HMMs. For each upper and lower case character and for frequent punctuation marks, such as space, colon, semi-colon and full stop, an individual HMM is built. Other, infrequent punctuation marks, such as exclamation or question marks, are mapped to a special garbage model. The collective garbage model is used because even in a large set of text lines there does not exist sufficient data to train such models individually.

The individual HMMs consist of 14 states that are connected in a linear topology. The output probability distribution is modeled by a mixture of Gaussian components. The character models are concatenated to word models, which share the individual character models. This allows us to share training data across words. While our approach is text-independent, we assume that each word in the text is included in the dictionary. A complete text line is modeled by concatenating these word models. We again refer to [18] for further details.

The HMMs are trained by applying the BaumWelch algorithm [23] using a training strategy proposed in [11]. First, a single Gaussian output distribution is used for each state. Each model is trained with four iterations. Then in a second step, the number of Gaussian mixture components is increased. This is accomplished by splitting the Gaussian distribution with the highest weight. The mean vectors of the two new Gaussian distributions are the mean of the original Gaussian \pm 0.2 times the standard deviation of the original distribution [33]. Then in the third step, we again train each model in four iterations using the new mixture components. Steps two and three are repeated until the desired number of Gaussian mixture components is reached. In the experiments described in this paper, the number of mixture components is increased up to a maximum of five Gaussians.

The system has been implemented using the HTK toolkit [33], originally developed for speech recognition. The toolkit employs the Baum-Welch algorithm for training and the Viterbi algorithm for recognition [23]. The output of the system is a transcription of the considered text line consisting of a sequence of words together with the log-likelihood score of each word. The score of a text line is the sum of the log-likelihood of all words. For both writer identification and verification only the output scores, but not the transcriptions are utilized.

\subsection{Writer identification}

For the task of writer identification, we present a text line of an unknown writer to each of the trained recognizers. Each recognizer outputs a transcription of the input text line together with its log-likelihood score. The log-likelihood scores are sorted in descending order. Using a confidence measure [20] defined on these log-likelihood scores enables us to implement a rejection mechanism. If the confidence measure of a text line is above a given threshold, the system returns the identity of the text line with the highest ranked score; otherwise the system rejects the input. Thus, if we have $n$-writers, the writer identification problem is an $n$-class classification problem with a reject option.

A very simple way to define a confidence measure, $\mathrm{cm}_{1}(t)$, for a text line $t$ is to use the log-likelihood score $l_{1}$ of the first ranked text line:

$\mathrm{cm}_{1}(t)=l_{1}$

A more elaborate confidence measure is inspired by the cohort score normalization technique used in the field of speaker verification $[1,24]$. The log-likelihood scores of the first $N$ ranks are used to calculate the confidence measure $\mathrm{cm}_{2}(t)$ of a text line $t$ as follows:

$\mathrm{cm}_{2}(t)=\frac{l_{1}-l_{\mathrm{avg}}}{|t|}$

where $|t|$ is the length of the text line $t$ in pixels and

$l_{\text {avg }}=\frac{1}{N} \sum_{j=2}^{N+1} l_{j}$

In (3) the sum of log-likelihoods is formed over the log-likelihoods of the competing $N$-best ranked writers. The index $j$ starts at 2 which requires that $N<n$. 


\subsection{Writer verification}

For the task of writer verification, the system must decide, based on some criterion, whether a text line with a claimed identity is in fact from this writer or whether it is an impostor attempt. We define the following verification criterion. If the confidence measure of a text line is above a certain threshold, we assume that the text line is in fact from the claimed writer; otherwise the input is classified as not being of the claimed identity. The writer verification problem is thus a two-class classification problem.

A very simple confidence measure is to use the loglikelihood score of the text line of the claimed identity, $l_{\text {claimed_identity, }}$ and define the confidence measure as

$\mathrm{cm}_{3}(t)=l_{\text {claimed_identity }}$

A more refined confidence measure can be defined by using the log-likelihood scores of the first $N$ ranks as follows:

$\mathrm{cm}_{4}(t)=\frac{l_{\text {claimed_identity }}-l_{\text {avg }}}{|t|}$

where $l_{\text {avg }}$ is given by

$l_{\mathrm{avg}}=\frac{1}{N} \sum_{\substack{j=1 \\ j \neq r(t)}}^{N+1} l_{j}$

The confidence measure in (5) is calculated from the difference of the log-likelihood score of the claimed identity and $l_{\text {avg }}$, and is normalized by the length of the text line. We calculate $l_{\text {avg }}$ based on the $N$-best ranked competing writers according to (6), where $r(t)$ is the rank of the claimed identity of text line $t$. Again, $N<n$ must hold.

\section{Experimental results}

\subsection{Database and experimental setup}

A verification system must decide whether a text line with a claimed identity was in fact written by that person or not. In the former case a person is called a client, in the latter case he or she is called an impostor [7]. Consequently, we have to define two sets. First, a client data set needs to be defined that contains text lines of writers that have to be accepted. This set is also used to test the system on the writer identification task. Second, we define an impostor data set that contains text lines of writers that have to be rejected. Impostor attempts can be divided into unskilled forgeries, where the impostor makes no effort to simulate a genuine handwriting, and skilled forgeries, where the impostor tries to imitate the handwriting of a client as closely as possible [22].

Our experiments are based on pages of handwritten text from the IAM database [19] $]^{1}$. The database currently contains over 1,500 pages of handwritten text written by over 650 different writers. Each page contains between five and eleven text lines. For each writer we use five pages of text. An example of such a page is shown in Fig. 4. To evaluate the performance of our system on the writer identification and verification tasks, the experimental setup consists of text lines from 100 clients, 20 unskilled impostors, and 20 skilled impostors.

The data set for the identification experiment contains 4,103 text lines from 100 different writers containing 20,391 word instances and 5,578 unique word classes.

We conducted two different writer verification experiments. The first verification experiment consists of data coming from clients and impostors using unskilled forgeries. The unskilled forgeries are obtained from the database by extracting 571 text lines produced by 20 writers. The writers of these text lines are disjoint from the 100 clients and no HMM recognizer exists that is trained on the handwriting of any of these 20 writers. Based on these text lines the impostor data set is constructed by assigning to each of these text lines seven identities of writers known to the system. The client data set is the same as the one used for the identification experiments. The rationale is that the number of text lines to be accepted is approximately the same as the number of text lines that have to be rejected. The impostor data set thus consists of $7 \times 571=3,997$ text lines. Overall, the complete data set consists of 8,100 text lines.

The second verification experiment is based on data coming from clients and impostors using skilled forgeries. The following protocol is used to obtain the skilled forgeries. A page of text written by an unknown author is presented to a person. The person is then asked to study and train the writing for $15 \mathrm{~min}$. When this time period has elapsed, the person is asked to copy the text onto a white sheet of paper where a ruler sheet is put below. An example of a skilled forgery of the text given in Fig. 4 is shown in Fig. 5.

\footnotetext{
${ }^{1}$ The database is publicly available at: http://www.iam.unibe.ch/ $\sim$ fki/iamDB
} 
The equipment takes two forms - an insulating guard on the jib of the crane, intended to prevent direct contact with the line, and electronic equipment with a sensitive probe mounted slightly forward of the head of the jib and with electronic assembly and warning apparatus in the driver's cab. Improved designs of both forms are being tried out at present.

Fig. 4 Example of a page of handwritten text

The test set for the second verification experiment is composed of two subsets. The client data set consists of one page of text from twenty different writers. A total of 169 text lines are extracted from these twenty pages. The same twenty pages are then skillfully forged. From these forgeries, another 169 text lines are extracted. Hence, in total 338 text lines are used.

For each writer the data set is split into four disjoint subsets, which enables us to perform full fourfold cross validation experiments. Iteratively, three out of the four sets are used to train the system and the remaining set is used to test its performance. Using cross validation guarantees that the training and test sets are disjoint, that our experiments are text independent, and

\section{The equipment takes two forms - an insulating guard on the jib of the crane, intendet to prevent direct contact with the line, and electronic equipment with a sensitive probe mounted slightly forward of the head of the jib and with electronic assembly and warning apparatus in the driver's cab. Improucd designs of both forms are being tried out at present.}

Fig. 5 Skilled forgery of the handwritten text shown in Fig. 4 that the maximum possible amount of test data is used. Of course, the impostor data set is used for testing only.

\subsection{Writer identification experiments}

In this experiment, a correct writer identification rate of $97.03 \%$ is achieved. In Fig. 6 the $n$-best list which measures the identification rate not only based on the first rank, but based on the first $n$ ranks is shown. The error rate drops from $2.97 \%$ to below $2 \%$ if the first six ranks are considered.

We use the confidence measures introduced in Sect. 3.2 to reject an input in case of uncertainty and calculate the corresponding error-rejection curves. As can be seen in Fig. 7, the $\mathrm{cm}_{1}(t)$ [see (1)] based system performs clearly inferior to the $\mathrm{cm}_{2}(t)$ [see (2)] based system. The best error-rejection curve is achieved using the confidence measure $\mathrm{cm}_{2}(t)$ with (3) and $N=1$. By rejecting $4.14 \%$ of the text lines with lowest confidence score, the error rate drops below $0.85 \%$ and if $21 \%$ of the text lines with lowest confidence score are rejected, the error rate drops below $0.1 \%$.

To investigate how our system scales with an increasing number of writers, we perform the following experiment. We start with text lines from ten writers and present them to the ten systems which are trained on these writers. We then increase the number of writers and systems by steps of ten up to hundred and measure the writer identification rate. The relationship between the identification rate and the number of writers is plotted in Fig. 8 for the case where only the best ranked system is considered $(n=1)$, and the case where the top five ranked systems are taken into account $(n=5)$. We observe that our system scales quite favorably with an increasing number of writers.

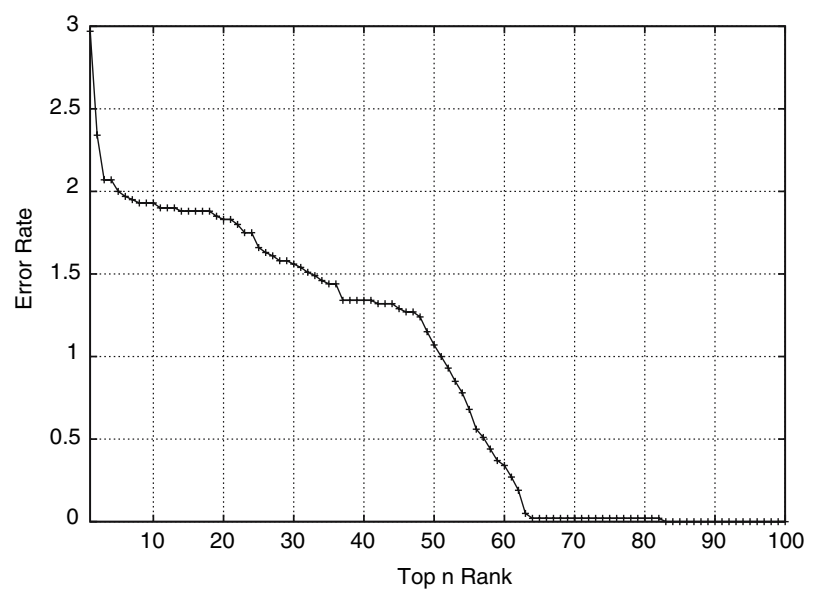

Fig. $6 \mathrm{~N}$-best list for the 100 writers identification experiment 


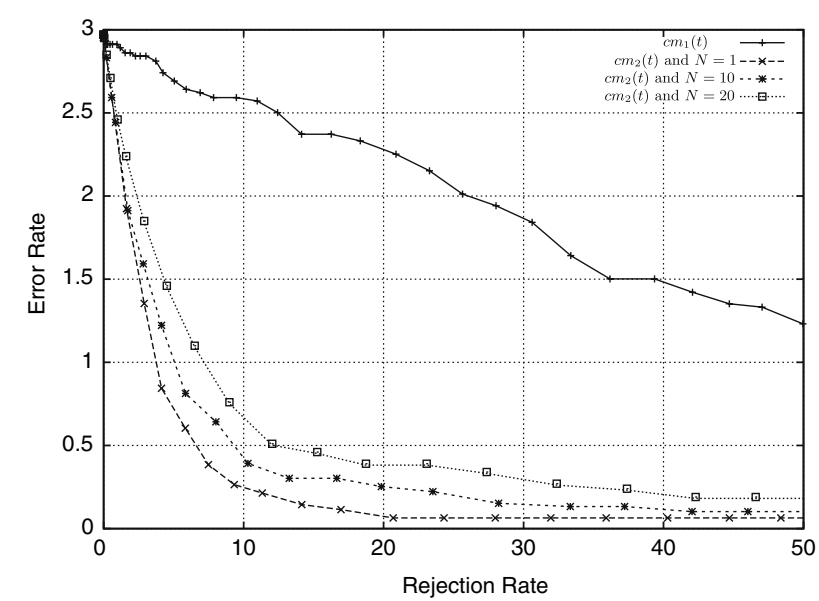

Fig. 7 Error-rejection curves for the 100 writers identification experiment

For example, if the number of writers is doubled from 50 to 100 then the writer identification rate decreases by only one percent for $n=1$ and by $0.2 \%$ for $n=5$.

\subsection{Writer verification experiments}

A verification system can make two types of errors. It can falsely reject a text line coming from a client, or it can falsely accept a text line coming from an impostor. The results of the verification experiments are presented in the form of Receiver Operator Characteristic (ROC) curves [7] using different confidence measures. To plot the ROC curves the minimal and the maximal confidence measure is calculated and then varied.

In the verification experiment using unskilled forgeries, the ROC curve based on $\mathrm{cm}_{3}(t)$ shows considerably worse performance then the ROC curves based on $\mathrm{cm}_{4}(t)$ (see Fig. 9). The best ROC curve is produced using the confidence measure based on (5) and (6). An Equal Error Rate (EER) of about 2.0\% is achieved. For $N=1$, a False Acceptance Rate (FAR) of $0.8 \%$ is obtained at a False Rejection Rate (FRR) of $3.4 \%$. Increasing the number of writers $N$ to calculate the confidence measures $\mathrm{cm}_{4}(t)$ does not

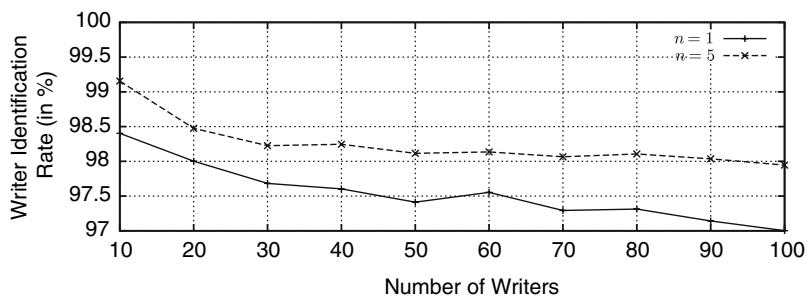

Fig. 8 Identification rate as a function of the number of writers

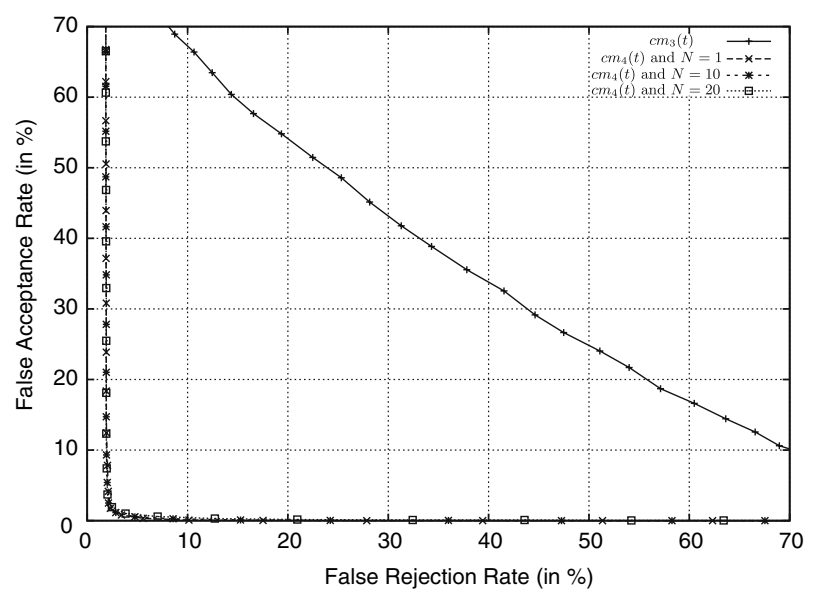

Fig. 9 ROC curves for the verification experiment using unskilled forgeries

lead to substantionally different ROC curves in this experimental setup.

The ROC curves for the verification experiment using skilled forgeries are given in Fig. 10. Again, the ROC curve based on $\mathrm{cm}_{4}(t)$ produces superior results compared to $\mathrm{cm}_{3}(t)$. The EER is approximately $4.47 \%$. Using $N=1$ to calculate the confidence measure produces a slightly better ROC curve when compared to using $N=10$ or $N=20$.

In Fig. 11 an example of the first type of error is shown. A text line coming from the same writer as the other three text lines is falsely rejected. The second type of error is shown in Fig. 12. A text line coming from an impostor is falsely assumed to come from the same writer who has written the three other text lines. In the second case, it is difficult even for a human observer to discriminate the two handwritings.

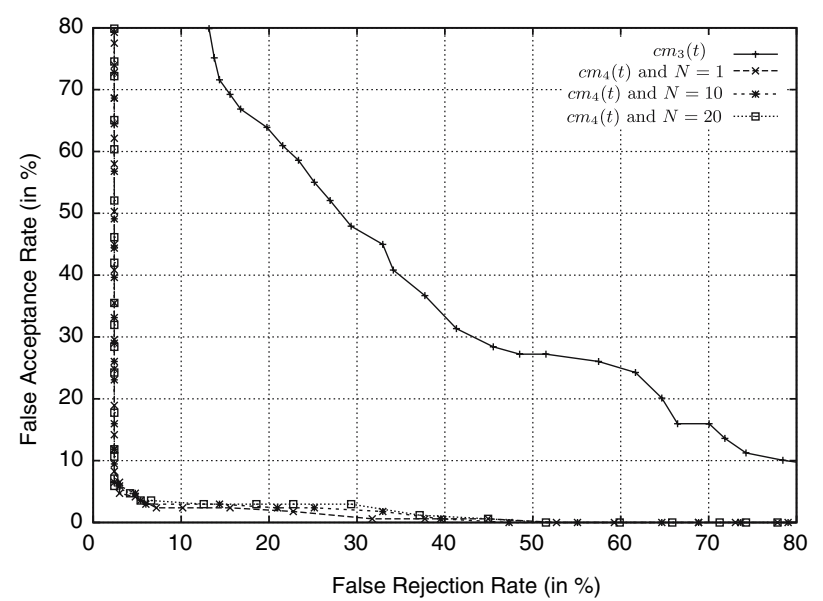

Fig. 10 ROC curves for the verification experiment using skilled forgeries 
He used to come to Pierre's Bar again and again. No be couldu't really remember him, he was one of the crowd. The Falsely rejected text line:

sun came out; Mart went shopping; I

Fig. 11 Example of false rejection error

\subsection{Writer Identification and Text Recognition Rate}

Our system uses HMM based handwriting recognizers which are optimized for text recognition and is based on the hypothesis that the word recognition rate is higher when the input comes from the writer whose data are used to train the system. We expect a large difference in text recognition performance between the case where the training and input data come from the same writer and the case where they come from different writers. In Table 1 we distinguish between the case where training and input data come from the same writer (row Same) and the case where they come from different writers (row Other). Obviously, there is a huge difference in word recognition performance between the two cases, which confirms our hypothesis.

In Fig. 13, the average text recognition rate per writer is shown on text lines coming from Writer 24 . The system that is trained with text lines from Writer 24 achieves an average text recognition rate of $78.76 \%$. In contrast, the text recognition rates of the systems which are trained with text lines from other writers achieve an average text recognition rate of only $14.07 \%$ in average. In Fig. 14 training samples of the four systems which achieve the highest text recognition rate in Fig. 13 are shown. Visually inspecting the text lines reveals that all four handwritings are indeed similar, especially those of Writers 5, 24, and 29. The letters are standing upright, are of similar width, and

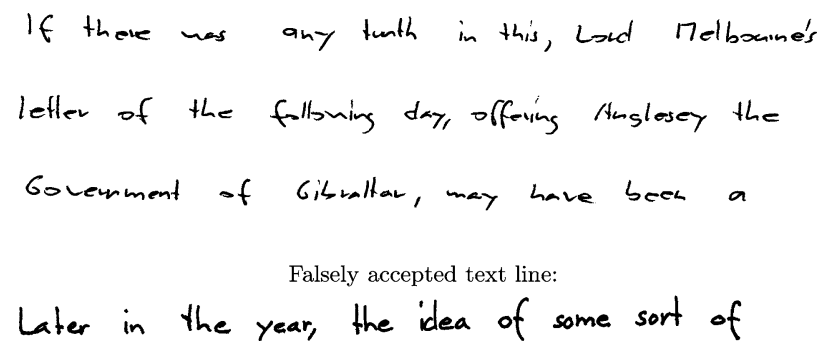

Fig. 12 Example of false acceptance error
Table 1 Word recognition rates for 100 writers identification experiment

\begin{tabular}{ll}
\hline & Word recognition rate (\%) \\
\hline Same & 63.12 \\
Other & 11.38 \\
\hline
\end{tabular}

the ascender, the middle, and the descender regions are of similar proportion. This confirms the feasibility of the approach proposed in this paper.

\section{Conclusion}

In this paper, we have presented a system that uses HMM based text line recognizers for the tasks of text independent off-line writer identification and verification. The basic input units presented to the system are handwritten text lines. From each text line, nine features are extracted. Using these features, we train a recognizer for each writer. A text line of unknown identity is presented to each of these recognizers. As output, each recognizer produces a transcription of the input text line with a log-likelihood score. Based on these scores a ranking in descending order is generated which is used for both identification and verification.

On the writer identification task, we achieve a correct identification rate of $97.03 \%$ in a 100 writers experiment using 4,103 lines of text. Experimenting with a set of confidence measures we show that by rejecting $4.14 \%$ of the text lines the error rate drops below $0.85 \%$ and a recognition rate of $99.9 \%$ is achieved by rejecting $21 \%$ of the text lines with the lowest confidence measure. Regarding writer verification, our system performs very well on both tasks of

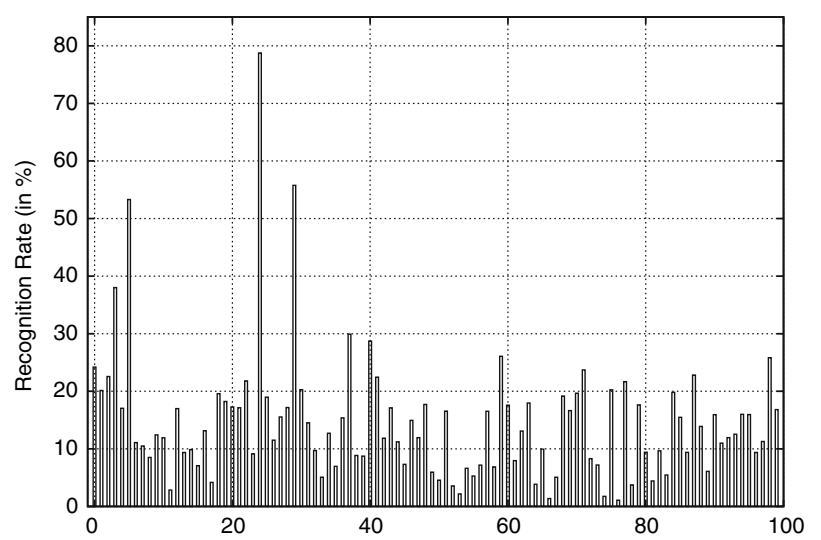

Fig. 13 Average text recognition rate for text lines coming from Writer 24 
Fig. 14 Training samples from the four systems which achieve the highest average text recognition rate for Writer 24 (see Fig. 13)

First ranked system (Writer 24)

By the end of the month he still delighted in Maples. He told Concurry that he enjoyed it as much as his health permitted him to enjoy Second ranked system (Writer 29)

When the sailing season was past, he sent Pearl back to England, and returned to Roure for the winter. In bak November, he was 'suffering as usual', but hopad, he told Third ranked system (Writer 5)

A pleasant life truly!... It so happens that I have an Ualian who is perhaps the best Valet de Chambre that ever was. But he has not one word of Enghish.' While he was Fourth ranked system (Writer 3)

$$
\begin{aligned}
& \text { What infatuation! Personally lam indifferent, but I really } \\
& \text { tremble for my country! I may be mistaken, } 1 \text { tho' } 1 \\
& \text { cannot but fear that the exasperation of the People }
\end{aligned}
$$

accepting clients and rejecting impostors. An Equal Error Rate of $2.0 \%$ is achieved on a total of 8,100 text lines coming from 100 clients and 20 impostors using unskilled forgeries. Using skilled forgeries, the Equal Error Rate raises to $4.47 \%$ on a test set that consists of 169 text lines from 20 clients and 169 text lines from 20 impostors.

While we have shown that our system scales from $n=10$ to $n=100$ writers, it is an open question how it would scale for a very large number of writers, e.g., $n>1000$. Performing such an experiment would require a significant extension of our database. One way to reduce training time and the amount of data needed from each writer is to train a general background model and then adapt this model to obtain a specific writer model. These issues are left for future work.

\section{Originality and contribution}

Hidden Markov Models (HMMs) have become a standard tool in handwriting recognition. HMM based recognizers have a number of advantages over other approaches. First, they are resistant to noise and can cope with shape variations. Second, they allow to model characters of variable width occurring in the text. Third, HMM based recognizers are able to implicitly segment a text line into words and characters, a task that is difficult to perform explicitly. Last, there exist standard algorithms for training and testing.

The main contribution of this paper is to apply HMMs to the problem of off-line, text independent writer identification and verification. To the best of our knowledge, HMMs have not been applied to these 
tasks before. Our system is text independent, i.e., any text can be used for writer identification and verification. Using text lines as basic input units, the proposed approach is positioned between the case where only a single word is used and systems that require a whole page of text as input. Single words carry little information and corresponding systems may not scale well with an increasing number of writers. On the other hand, requiring a whole page of text being available may be too restrictive for certain applications. Consequently, the approach proposed in this paper may provide a good trade-off between scalability and performance on the one hand, and user-friendliness and flexibility on the other hand.

Experiments using text lines from 100 writers on the identification and two set of experiments on the verification task show that our approach performs very well. Possible applications of the system are in forensic writer identification, the retrieval of handwritten documents from a database, or authorship determination of historical manuscripts.

Acknowledgments This work is supported by the Swiss National Science Foundation program "Interactive Multimodal Information Management (IM2)" in the Individual Project "Access and Content Protection (ACP)", as part of NCCR. The authors also thank Dr. Urs-Victor Marti and Dr. Matthias Zimmermann for providing the recognition system and the IAMDatabase, and Dr. Simon Günter for valuable comments and advice. Furthermore, we would like to thank the anonymous reviewers for their valuable comments.

\section{References}

1. Ariyaeeinia AM, Sivakumaran P (1997) Analysis and comparison of score normalization methods for text-dependent speaker verification. In: Proceedings of the 5th European conference on speech communication and technology, pp 1379-1382

2. Baird HS (2003) Digital libraries and document image analysis. In: Proceedings of the 7th international conference on document analysis and recognition, pp 2-14

3. Baird HS, Govindaraju V (eds) (2004) Proceedings of the first international workshop on document image analysis for libraries. IEEE Computer Society

4. Bensefia A, Nosary A, Paquet T, Heutte L (2002) Writer identification by writer's invariants. In: Proceedings of the international workshop on frontiers in handwriting recognition, pp 274-279

5. Bensefia AT, Paquet T, Heutte L (2003) Information retrieval based writer identification. In: Proceedings of the 7th international conference on document analysis and recognition, pp 946-950

6. Bensefia A, Paquet T, Heutte L (2004) Handwriting analysis for writer verification. In: Proceedings of the 9th international workshop on frontiers in handwriting recognition, pp 196-201

7. Bimbot F, Chollet G (1997) Assessement of speaker verification systems. In: Gibbon D, Moore R, Winski R (eds)
Handbook of standards and resources for spoken language systems. Mouton de Gruyter, pp 408-480

8. Bulacu M, Schomaker L, Vuurpijl L (2003) Writer identification using edge-based directional features. In: Proceedings of the 7th international conference on document analysis and recognition, pp 937-941

9. Bunke H (2003) Recognition of cursive Roman handwritingpast, present and future. In: Proceedings of the 7th international conference on document analysis and recognition, pp 448-461

10. Cha SH, Srihari S (2000) Multiple feature integration for writer verification. In: Proceedings of the 7th international workshop on frontiers in handwriting recognition, pp 333342

11. Günter S, Bunke H (2004) HMM-based handwritten word recognition: on the optimization of the number of states, training iterations and Gaussian components. Pattern Recogn 37:2069-2079

12. Hertel C, Bunke H (2003) A set of novel features for writer identification. In: Kittler J, Nixon M (eds) Audio-and videobased biometric person authentication, pp 679-687

13. Jain A, Hong L, Pankanti S (2000) Biometric identification. Commun ACM 43:91-98

14. Jain AK, Bolle R, Pankanti S (eds) (2002) Biometrics-personal identification in networked society. Springer, Berlin Heidelberg New York

15. Kittler J, Nixon MS (eds) (2003) Audio- and video-based biometric person authentication. Springer, Berlin Heidelberg New York

16. Leclerc F, Plamondon R (1994) Automatic signature verification: the state of the art 1989-1993. In: Plamondon R (ed) Progress in automatic signature verification. World Scientific Publication Co, Singapore, pp 13-19

17. Leedham G, Chachra S (2003) Writer identification using innovative binarised features of handwritten numerals. In: Proceedings of the 7th international conference on document analysis and recognition, pp 413-417

18. Marti U-V, Bunke H (2001) Using a statistical language model to improve the performance of an HMM-based cursive handwriting recognition system. Int J Pattern Recogn Artif Intell 15:65-90

19. Marti U-V, Bunke H (2002) The IAM-database: an English sentence database for off-line handwriting recognition. Int $\mathbf{J}$ Doc Anal Recogn 5:39-46

20. Marukatat S, Artières T, Gallinari P, Dorizzi B (2002) Rejection measures for handwriting sentence recognition. In: Proceedings of the 8th international conference on frontiers in handwriting recognition, pp 25-29

21. Nosary A, Heutte L, Paquet T, Lecourtier Y (1999) Defining writer's invariants to adapt the recognition task. In: Proceedings of the 5th international conference on document analysis and recognition, pp 765-768

22. Plamondon R, Lorette G (1989) Automatic signature verification and writer identification-the state of the art. In Pattern Recognition, vol 22, pp 107-131

23. Rabiner LR (1989) A tutorial on hidden Markov models and selected applications in speech recognition. In: Proceedings of the IEEE, vol 77, pp 257-285

24. Rosenberg AE, Delong J, Huang CH, Soong FK (1992) The use of cohort normalized scores for speaker verification. In: Proceedings of the international conference on spoken language processing, pp 599-602

25. Said HES, Tan T, Baker K (2000) Personal identification based on handwriting. Pattern Recogn 33:149-160

26. Schlapbach A, Bunke H (2004) Off-line handwriting identification using HMM based recognizers. In: Proceedings of 
the 17 th international conference on pattern recognition, vol 2, pp 654-658

27. Schlapbach A, Bunke H (2004) Using HMM based recognizers for writer identification and verification. In: Proceedings of the 9th international workshop on frontiers in handwriting recognition, pp 167-172

28. Schlapbach A, Bunke H (2005) Writer identification using an HMM-based handwriting recognition system: to normalize the input or not? In: Proceedings of the 12th conference of the international graphonomics society, pp 138-142

29. Schomaker L, Bulacu M (2004) Automatic writer identification using connected-component contours and edge-based features of uppercase western script. IEEE Trans Pattern Anal Mac Intell 26:787-798

30. Schomaker L, Bulacu M, Franke K (2004) Automatic writer identification using fragmented connected-component contours. In: Proceedings of the 9th international workshop on frontiers in handwriting recognition, pp 185-190

31. Srihari S, Shi Z (2004) Forensic handwritten document retrieval system. In: Proceedings of the first international workshop on document image analysis for libraries, pp 188-194

32. Steinherz T, Rivlin E, Intrator N (1999) Off-line cursive script word recognition-a survey. Int J Doc Anal Recogn 2:90-110

33. Young S, Evermann G, Kershaw D, Moore G, Odell J, Ollason D, Povey D, Valtchev V, Woodland P (2002) The HTK Book

34. Zhang B, Srihari SN, Lee S (2003) Individuality of handwritten characters. In: Proceedings of the 7 th international conference on document analysis and recognition, vol 7 , pp 1086-1090

35. EN Zois, Anastassopoulos V (2000) Morphological waveform coding for writer identification. Pattern Recogn 33:385398

\section{Author Biographies}

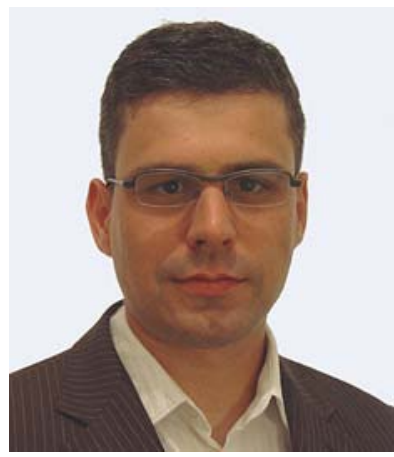

Andreas Schlapbach received his M.S. degree in Computer Science from the University of Bern, Switzerland, in 2003. He is currently a Ph.D. student at the Institute of Computer Science and Applied Mathematics at the University of Bern. His current research interests include writer identification and verification and feature extraction and selection techniques.

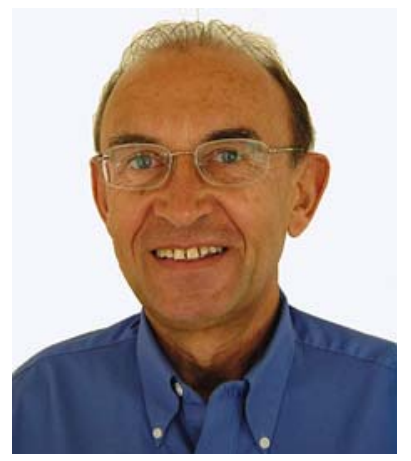

Horst Bunke received his M.S. and Ph.D. degrees in Computer Science from the University of Erlangen, Germany. In 1984, he joined the University of Bern, Switzerland, where he is a professor in the Computer Science Department. He was Department Chairman from 19921996, Dean of the Faculty of Science from 1997 to 1998, and a member of the Executive Committee of the Faculty of Science from 2001 to 2003. From 1998 to 2000 Horst Bunke served as 1st Vice-President of the International Association for Pattern Recognition (IAPR). In 2000 he also was Acting President of this organization. Horst Bunke is a Fellow of the IAPR, former Editor-in-Charge of the International Journal of Pattern Recognition and Artificial Intelligence, Editor-in-Chief of the journal Electronic Letters of Computer Vision and Image Analysis, Editor-in-Chief of the book series on Machine Perception and Artificial Intelligence by World Scientific Publ. Co., Advisory Editor of Pattern Recognition, Associate Editor of Acta Cybernetica, and Former Associate Editor of the International Journal of Document Analysis and Recognition, and Pattern Analysis and Applications. Horst Bunke held visiting positions at the IBM Los Angeles Scientific Center (1989), the University of Szeged, Hungary (1991), the University of South Florida at Tampa (1991, 1996, 1998-2006), the University of Nevada at Las Vegas (1994), Kagawa University, Takamatsu, Japan (1995), Curtin University, Perth, Australia (1999), and Australian National University, Canberra (2005). He served as a co-chair of the 4th International Conference on Document Analysis and Recognition held in Ulm, Germany, 1997 and as a Track Co-Chair of the 16th and 17th International Conference on Pattern Recognition held in Quebec City, Canada and Cambridge, UK in 2002 and 2004, respectively. Also he was chairman of the IAPR TC2 Workshop on Syntactic and Structural Pattern Recognition held in Bern 1992, a co-chair of the 7th IAPR Workshop on Document Analysis Systems held in Nelson, NZ, 2006, and a co-chair of the 10th Int. Workshop on Frontiers in Handwriting Recognition, held in La Baule, France, 2006. Horst Bunke was on the program and organization committee of many other conferences and served as a referee for numerous journals and scientific organizations. $\mathrm{He}$ is on the Scientific Advisory Board of the German Research Center for Artificial Intelligence (DFKI). Horst Bunke has more than 500 publications, including 34 authored, co-authored, edited or co-edited books and special editions of journals. 